\title{
Avaliação da associação entre qualificação de médicos e enfermeiros em atenção primária em saúde e qualidade da atenção
}

Claudia Danyella Alves Leão ${ }^{1}$

Antônio Prates Caldeira ${ }^{2}$

${ }^{1}$ Universidade Estadual de Montes Claros

${ }^{2}$ Departamento de Saúde da Mulher e da Criança, Universidade Estadual de Montes Claros. Rua Monte Pascoal 225, Ibitutuna. 39.401-347 Montes Claros MG.

antonio.caldeira@unimontes.br

\author{
Assessment of the association between the qualification \\ of physicians and nurses in primary healthcare \\ and the quality of care
}




\section{Introdução}

Desde a Conferência Mundial de Saúde em AlmaAta, o fortalecimento da atenção primária vem sendo adotado em diversos países como estratégia de organização do sistema de saúde e otimização dos recursos disponíveis ${ }^{1}$. O Brasil, após a implantação do Sistema Único de Saúde (SUS) definiu a atenção primaria à saúde (APS) como porta de entrada, criando, alguns anos mais tarde, o Programa de Saúde da Família como principal estratégia para alcançar a organização desejada ${ }^{2}$. Os gestores municipais de saúde aderiram rapidamente à Estratégia Saúde da Família (ESF) como proposta de organizar a APS, o que pode ser confirmado pelo crescimento significativo do número de equipes em todo país ${ }^{3}$. Não é possível afirmar que esse aumento seja decorrente de adesão ideológica à proposta de reorganização do modelo. Até o momento, o aumento do número de equipes de saúde da família não tem garantido a mudança do modelo de saúde hegemônico, que valoriza as praticas médicas, curativistas, especializadas e hospitalocêntricas. Para alguns autores, esse fato decorre da falta de profissionais adequadamente preparados para lidar com as novas atribuições exigidas pelo novo modelo que enfatiza a $\mathrm{APS}^{4,5}$.

Segundo Starfield ${ }^{6}$, a efetivação da APS se dá por meio dos seus atributos norteadores. A autora sistematizou o conceito da APS através de atributos denominados essenciais (primeiro contato, longitudinalidade, integralidade da atenção e coordenação do cuidado) e derivados (atenção centrada na família e orientação comunitária). A persistência do modelo biomédico e a lacuna que se observa sobre a adesão aos atributos da APS tornam imprescindível a discussão sobre a formação de recursos humanos para o SUS, buscando encontrar as melhores alternativas para garantir que as práticas profissionais atendam aos desafios necessários para a implementação de uma APS de qualidade.

Apesar da relevância do profissional bem formado para a consolidação do novo modelo de assistência, a literatura nacional ainda registra poucos estudos na área. Existe, pois, a necessidade de se conhecer o impacto da formação e da qualificação profissional sobre o desenvolvimento dos atributos da APS. Já se sabe que os serviços de saúde que se organizam a partir de uma APS estruturada em conformidade com os seus atributos ordenadores são mais efetivos e de melhor qualidade ${ }^{7-10}$. As mudanças nos cursos de graduação, na tentativa de formar profissionais mais adequados para a assistência na APS são lentas e heterogêneas, pois não acontecem da mesma forma em todas as instituições ${ }^{11}$. O crescimento acelerado da ESF parece de fato exigir uma formação no âmbito de pós-graduação para o exercício competente das práticas usuais dessa área.

Existem três tipos de pós-graduações lato sensu. A residência médica, que é exclusiva para o profissional médico, os cursos de especialização abertos a vários cursos de saúde e as residências multiprofissionais para a especialização dos diferentes trabalhadores de nível superior da área da saúde ${ }^{12}$. De acordo com Gil ${ }^{13}$, as experiências promovidas pelas residências podem representar a melhor oportunidade para uma reflexão em torno de alternativas que permitam rever os caminhos para a formação dos profissionais, na perspectiva de um trabalho mais coerente com o que se propõe a ESF. As especializações na modalidade de residência foram concebidas para dar suporte teórico-prático aos profissionais já inseridos nas equipes e oferecer, em especial, aos egressos dos cursos de medicina e enfermagem, uma formação mais voltada às necessidades da $\operatorname{ESF}^{13}$.

No presente estudo, objetivou-se verificar a associação entre os atributos da APS e a qualificação profissional promovida pela Residência de Medicina de Família e Comunidade e pela Residência Multiprofissional em Saúde da Família.

\section{Metodologia}

Trata-se de um estudo transversal, descritivo, analítico, de abordagem quantitativa. O trabalho foi conduzido em Montes Claros, ao Norte do Estado de Minas Gerais no primeiro trimestre de 2010. A cidade possui população estimada em mais de 360 mil pessoas. Quanto aos serviços de atenção primária, o município contava à época da coleta de dados com 49 equipes de saúde da família (44 localizados na zona urbana e cinco na zona rural), 15 PACS (Programa Agentes Comunitários de Saúde) e 10 centros de saúde, sendo estes responsáveis pela parte da população que não possui cobertura da ESF.

É relevante ressaltar a presença, no município, do Programa Integrado de Residência em Medicina de Família e Comunidade/Curso de Especialização na modalidade Residência em Saúde da Família para Enfermeiro e Cirurgião-Dentista. Trata-se de uma parceria entre o Ministério da Saúde, a Universidade Estadual de Montes Claros e a Prefeitura Municipal/Secretária Municipal de Saúde voltada à qualificação profissio- 
nal para o trabalho no campo dos cuidados primários em saúde.

Buscou-se verificar os atributos da APS em equipes com profissionais qualificados e sem qualificação específica, através da utilização do Instrumento de Avaliação da Atenção Primária (Primary Care Assessment Tool - PCA-Tool) desenvolvido nos Estados Unidos por Starfield e já validado no Brasil ${ }^{14}$. O instrumento possui questões distintas para aferição dos atributos da APS a partir do cuidado ao adulto e à criança. Para este estudo, optou-se pela utilização das questões referentes ao cuidado infantil, compreendendo-se que a atenção à saúde infantil pode ser mais reveladora da organização dos serviços de APS, por incluir maior número de ações de promoção à saúde e prevenção de doenças. Assim, para a coleta de dados e verificação dos escores dos atributos, a população alvo deste estudo foi constituída pelos cuidadores de crianças de zero a dois anos de idade, residentes e cadastradas nas áreas de abrangência das equipes da ESF do município. Foram incluídas apenas as equipes de saúde da ESF localizadas na zona urbana, que estavam em funcionamento há pelo menos um ano do início da coleta de dados. O critério de inclusão das equipes apenas da área urbana foi estabelecido por limitações logísticas. A determinação de se incluir equipes ativas há mais de um ano objetivou a incorporação de dados referentes a um maior contato temporal entre a população e os serviços de saúde. Após aplicação dos critérios apresentados a população identificada foi de 3.647 famílias (com crianças na referida faixa etária), distribuídas em 43 das equipes (uma equipe foi excluída porque havia sido implantada há menos de um ano e ainda estava incompleta). O processo amostral foi aleatório, casual simples, com identificação das famílias a partir do cadastro das mesmas nas unidades da ESF. O cálculo amostral definiu a necessidade de alocação de 350 famílias, considerando-se um erro amostral de 5\% e nível de confiança de 95\%, a partir de uma prevalência do evento estudado de 50\% (utilização do serviço de saúde). Esse valor é habitualmente tomado como uma estimativa conservadora, pois aumenta o número da amostra.

A verificação da extensão dos atributos da APS para os serviços foi realizada através de entrevistas com os cuidadores nos próprios domicílios. O formulário aplicado (PCA-Tool infantil) é composto de 45 perguntas relacionadas aos atributos da APS (seis para avaliar acesso de primeiro contato, 10 para continuidade ou longitudinalidade, cinco para coordenação, 14 para integralidade, seis para orientação familiar e qua- tro para orientação comunitária), além de três perguntas iniciais que medem o grau de afiliação ao serviço de saúde. As respostas são do tipo Likert, com intervalo de um a quatro para cada atributo. Também foram coletados dados sobre características demográficas, socioeconômicas das crianças selecionadas. O instrumento foi aplicado por duas enfermeiras especialmente treinadas para uso do PCA-Tool.

A definição do serviço de referência e do grau de afiliação ao serviço foi realizada segundo orientações do instrumento utilizado. Posteriormente, os serviços referidos foram dicotomizados em "saúde da família" (para os cuidadores que referiam à ESF) e "outros serviços" (para os cuidadores que referiam serviço que não fosse à ESF).

Após processamento dos dados, foram calculados os escores de cada atributo, do essencial (obtido através da média dos atributos essenciais e do grau de afiliação), do derivado (obtido através da média dos atributos derivados) e do geral da APS (valor médio dos atributos essenciais e derivados e o do grau de afiliação). Os valores obtidos para cada escore na escala Likert foram convertidos para uma escala entre zero e 10 , onde escores com valores iguais ou maiores que 6,6 indicam uma extensão adequada de cada atributo ${ }^{15}$.

Para a comparação entre as equipes com residência (médicos e/ou enfermeiros habilitados pela residência) e sem residência (nenhum médico ou enfermeiro habilitado pela residência), as médias dos escores foram analisadas através do teste " $t$ " de Student e para análise das proporções utilizou-se do teste do qui-quadrado de Pearson. O nível de significância assumido foi de 5\% $(\mathrm{p}<0,05)$. Buscando associação entre o grau de orientação para a APS e as características socioeconômicas e demográficas, bem como as características profissionais e das unidades de saúde ajustou-se um modelo de regressão de Poisson, sendo estimada a razão de prevalências e o intervalo de confiança de 95\%. O software empregado foi o SPSS for windows versão 15.0.

As entrevistas foram conduzidas com os principais cuidadores, definidos como os adultos que passavam mais tempo com a criança e que eram responsáveis pelo provimento de atenção à saúde da criança. A coleta de dados com os familiares foi conduzida dentro dos padrões éticos preconizados, após assinatura do termo de consentimento livre e esclarecido. Foram observadas as recomendações da Resolução 196/96 do Conselho Nacional de Ética em Pesquisa e o estudo foi aprovado pelo Comitê de Ética em Pesquisas da Universidade Estadual de Montes Claros. 


\section{Resultados}

As equipes da ESF envolvidas no estudo tinham em média 5,7 anos $(\mathrm{DP}=3,2)$ de funcionamento. O tempo médio de permanência do profissional médico na equipe até o momento de realização do estudo foi de 1,8 anos $(\mathrm{DP} \pm 1,3)$ e do enfermeiro foi de 2,7 anos $(\mathrm{DP} \pm 2,0)$. Sobre a formação dos profissionais registrou-se que 20 médicos $(46,5 \%)$ possuíam a residência de medicina de família e comunidade e 22 enfermeiros $(51,2, \%)$ a residência multiprofissional em saúde da família.

Os principais cuidadores referidos pelas famílias foram as mães $(88,6 \%)$ e as avós $(5,1 \%)$. Das crianças selecionadas, 177 (50,6\%) eram do sexo feminino. A média de idade do grupo estudado foi de 12,7 meses (DP $\pm 6,7$ meses) e 213 $(60,9 \%)$ crianças possuem irmãos, sendo que destas $101(28,9 \%)$ possuem dois ou mais irmãos.

Observou-se que $272(77,7 \%)$ dos entrevistados identificaram a ESF como fonte regular de atenção para o cuidado de saúde das crianças enquanto $78(22,3 \%)$ consideraram outros serviços, apesar de serem cadastrados em equipes da ESF. Para as famílias que utilizavam a ESF como fonte regular de cuidado para as crianças o escore geral foi 6,4 (DP $\pm 1,2)$ enquanto que para as demais este escore foi igual a 5,7 (DP $\pm 1,1)$, registrando-se uma diferença estatisticamente significante entre os dois grupos ( $\mathrm{p}=0,000$ ).

A Tabelal expressa os escores dos atributos da APS, aferidos a partir da percepção dos cuidadores entrevistados das crianças que tem a ESF como fonte regular de atenção. Estes escores foram comparados entre as equipes onde os profissionais possuíam a Residência de Medicina e Comunidade ou a Residência Multiprofissional em Saúde da Família, com aquelas equipes onde os profissionais não tinham esta formação. Entre as equipes que participaram do estudo, 18 possuíam médicos e enfermeiros com residência, duas possuíam apenas o médico com residência e quatro possuíam apenas o enfermeiro com residência. As demais 19 equipes não possuíam nenhum profissional com formação em saúde da família na modalidade residência médica ou multiprofissional. Os escores observados mostram que em geral, as equipes com residência apresentam escores maiores para a maioria dos atributos avaliados e com diferença estatisticamente significante para os atributos da longitudinalidade, da integralidade de serviços básicos disponíveis, da integralidade de serviços complementares disponíveis, bem como para os escores essencial e geral.

Durante a análise dos dados, buscou-se identificar as variáveis que poderiam estar relacionadas com uma melhor avaliação dos cuidados percebidos pelo serviço, identificado como fonte regular de atenção para o cuidado de saúde das crianças. Nesse sentido, o grupo foi dicotomizado não em relação ao serviço preferencial, mas em relação ao escore atribuído a este serviço. Tal análise foi realizada através do Alto Escore Geral da APS ( $\left.\geq^{\wedge} 6,6\right)$, entre todas as crianças. A Tabela 2 mostra os resultados dessa análise. A partir

Tabela 1. Média dos escores para os atributos da APS, segundo os cuidadores das crianças que tem a ESF como fonte regular de atenção. Montes Claros (MG), 2009.

\begin{tabular}{|c|c|c|c|c|}
\hline Atributos da APS & $\begin{array}{l}\text { ESFprofissional } \\
\text { com residência } \\
(\text { Média e DP })\end{array}$ & $\begin{array}{l}\text { ESFprofissional } \\
\text { sem residência } \\
(\text { Média e DP) }\end{array}$ & $\begin{array}{l}\text { Teste } \\
\text { “t”" }\end{array}$ & $\begin{array}{c}\text { p- } \\
\text { valor }^{*}\end{array}$ \\
\hline Primeiro contato & $5,5 \pm 1,8$ & $5,4 \pm 1,9$ & 0,241 & 0,810 \\
\hline Longitudinalidade & $8,3 \pm 1,4$ & $7,9 \pm 1,8$ & 1,972 & $0,049^{\#}$ \\
\hline Coordenação & $5,9 \pm 3,3$ & $6,7 \pm 3,6$ & 1,002 & 0,320 \\
\hline Integralidade - serviços básicos disponíveis & $6,0 \pm 3,4$ & $4,9 \pm 3,7$ & 2,464 & $0,015^{\#}$ \\
\hline Integralidade - serviços complementares disponíveis & $5,1 \pm 1,7$ & $4,6 \pm 2,1$ & 2,112 & $0,036^{\#}$ \\
\hline $\begin{array}{l}\text { Integralidade - ações de promoção e prevenção } \\
\text { recebidos }\end{array}$ & $8,0 \pm 2,8$ & $7,9 \pm 2,8$ & 0,264 & 0,792 \\
\hline Orientação familiar & $4,2 \pm 2,7$ & $4,4 \pm 2,5$ & 0,698 & 0,485 \\
\hline Orientação comunitária & $5,8 \pm 2,5$ & $5,3 \pm 2,5$ & 1,365 & 0,173 \\
\hline Essencial & $7,0 \pm 1,0$ & $6,6 \pm 1,3$ & 2,852 & $0,005^{\#}$ \\
\hline Derivado & $5,1 \pm 2,2$ & $4,8 \pm 2,0$ & 1,231 & 0,219 \\
\hline Geral & $6,6 \pm 1,1$ & $6,2 \pm 1,3$ & 2,626 & $0,009^{\#}$ \\
\hline
\end{tabular}

${ }^{*}$ Diferença estatisticamente significativa $(\mathrm{p}<0,05)$. 
destes resultados e tomando-se todas as variáveis com nível de significância de até $25 \%(\mathrm{p}<0,25)$, realizou-se uma análise de regressão múltipla (Regressão de Poisson) para definir um modelo explicativo final. Permaneceram no modelo final como variáveis associadas com alto valor geral da APS o serviço preferencial da ESF, o número de atendimentos maior que dez e a qualificação do profissional que assiste a criança (Tabela 3 ).

Para uma análise específica das crianças cujos cuidadores referiam a ESF como fonte regular de atenção, buscou-se conhecer as características que pudessem estar associadas com melhor avaliação da estratégia, através do Alto Escore Geral da APS (Tabela 4). Novamente, a partir dos resultados dessa tabela e tomando-se todas as variáveis com nível de significância de até $25 \%$ $(\mathrm{p}<0,25)$, realizou-se a análise de regressão múl- tipla (Regressão de Poisson) para definir um modelo explicativo final. Permaneceram neste

Tabela 3. Modelo ajustado do Alto Valor Geral da APS. Montes Claros, MG, 2009

\begin{tabular}{lcr}
\hline \multicolumn{1}{c}{ Variáveis } & RP (IC 95\%) & p-valor $^{*}$ \\
\hline $\begin{array}{l}\text { Serviço preferencial } \\
\quad \text { Outros }\end{array}$ & 1 & 0,000 \\
$\quad$ Saúde da Família & $1,22(1,10-1,36)$ & \\
Numero de atendimentos & & 0,023 \\
$\quad \leq 10$ & 1 & \\
$\quad>10$ & $1,13(1,02-1,25)$ & \\
Profissional com residência & 1 & 0,007 \\
$\quad$ Não & $1,15(1,04-1,27)$ & \\
$\quad$ Sim &
\end{tabular}

Tabela 2. Características sociodemográficas e de utilização dos serviços de saúde classificados com Alto e Baixo Escore Geral da APS. Montes Claros (MG), 2009.

\begin{tabular}{|c|c|c|c|}
\hline Variáveis & $\begin{array}{c}\text { Alto Escore Geral } \\
\text { da APS }(\geq 6,6) \\
n(\%)\end{array}$ & $\begin{array}{c}\text { Baixo Escore Geral } \\
\text { da APS }(\geq 6,6) \\
n(\%)\end{array}$ & p-valor ${ }^{*}$ \\
\hline Idade das crianças (meses) & & & 0,793 \\
\hline$<12$ & $66(47,1)$ & $96(45,7)$ & \\
\hline $12-23$ & $74(52,9)$ & $114(54,3)$ & \\
\hline Idade das mães (anos) & & & 0,194 \\
\hline$\leq 25$ & $78(55,7)$ & $98(46,9)$ & \\
\hline$>25$ & $62(44,3)$ & $111(53,1)$ & \\
\hline Idade dos pais (anos) & & & 0,543 \\
\hline$\leq 25$ & $46(33,3)$ & $61(29,9)$ & \\
\hline$>25$ & $92(66,7)$ & $143(70,1)$ & \\
\hline Cuidador principal & & & 0,493 \\
\hline Mãe & $126(90)$ & $184(87,6)$ & \\
\hline Outros & $14(10)$ & $26(12,4)$ & \\
\hline Profissão do pai & & & 0,224 \\
\hline Qualificado & $95(70,9)$ & $131(64,5)$ & \\
\hline Não qualificado & $39(29,1)$ & $72(35,5)$ & \\
\hline Escolaridade materna & & & 0,051 \\
\hline$\leq 8$ anos & $59(42,2)$ & $111(53,1)$ & \\
\hline$>8$ anos & $80(57,6)$ & $98(46,9)$ & \\
\hline Escolaridade paterna & & & 0,149 \\
\hline$\leq 8$ anos & $64(49,2)$ & $113(57,4)$ & \\
\hline$>8$ anos & $66(50,8)$ & $84(42,6)$ & \\
\hline Serviço fonte regular de atenção & & & 0,000 \# \\
\hline Saúde da Família & $122(87,1)$ & $150(71,4)$ & \\
\hline Outros & $18(12,9)$ & $60(28,6)$ & \\
\hline No de consultas realizadas no local & & & 0,020 \# \\
\hline$\leq 10$ vezes & $76(54,3)$ & $140(66,7)$ & \\
\hline$>10$ vezes & $64(45,7)$ & $70(33,3)$ & \\
\hline Qualificação profissional (residência em saúde da família) & & & 0,007 \# \\
\hline Sim & $101(72,1)$ & $122(58,1)$ & \\
\hline Não & $39(27,9)$ & $88(41,9)$ & \\
\hline
\end{tabular}

" Teste do qui-quadrado. ${ }^{*}$ Diferença estatisticamente significativa $(\mathrm{p}<0,05)$. 
Tabela 4. Características sociodemográficas e de utilização dos serviços de saúde classificados com Alto e Baixo Escore Geral da APS entre as crianças que tem a ESF como fonte regular de atenção à saúde. Montes Claros (MG), 2009.

\begin{tabular}{|c|c|c|c|}
\hline Variáveis & $\begin{array}{c}\text { Alto Escore Geral } \\
\text { da APS }(\geq 6,6) \\
\text { n }(\%)\end{array}$ & $\begin{array}{c}\text { Baixo Escore Geral } \\
\text { da APS }(\geq 6,6) \\
n(\%)\end{array}$ & p-valor ${ }^{*}$ \\
\hline Idade das crianças (meses) & & & 0,636 \\
\hline$<12$ & $58(47,5)$ & $67(44,7)$ & \\
\hline $12-23$ & $64(52,5)$ & $83(55,3)$ & \\
\hline Idade das mães (anos) & & & 0,577 \\
\hline$\leq 25$ & $67(54,9)$ & $77(51,7)$ & \\
\hline$>25$ & $55(45,1)$ & $72(48,3)$ & \\
\hline Idade dos pais (anos) & $42(35,0)$ & $46(31,7)$ & 0,580 \\
\hline$\leq 25$ & $78(65,0)$ & $99(68,3)$ & \\
\hline$>25$ & & & \\
\hline Cuidador principal & & & 0,859 \\
\hline Mãe & $109(89,3)$ & $133(88,7)$ & \\
\hline Outros & $13(11,3)$ & $17(10,7)$ & \\
\hline Profissão do pai & & & 0,101 \\
\hline Qualificado & $82(67,2)$ & $86(57,3)$ & \\
\hline Não qualificado & $36(29,5)$ & $58(38,7)$ & \\
\hline Não informado & $4(3,3)$ & $6(4,0)$ & \\
\hline Profissão da mãe & & & 0,141 \\
\hline Qualificado & $30(24,6 \%)$ & $26(17,3)$ & \\
\hline Não qualificado & $92(75,4)$ & $124(82,7)$ & \\
\hline Não informado & 0 & 0 & \\
\hline Escolaridade materna & & & $0,030^{\#}$ \\
\hline$\leq 8$ anos & $55(45,1)$ & $88(58,7)$ & \\
\hline$>8$ anos & $66(54,1)$ & $62(41,3)$ & \\
\hline Não informado & $1(0,8)$ & 0 & \\
\hline Escolaridade paterna & & & 0,119 \\
\hline$\leq 8$ anos & $58(47,5)$ & $85(56,7)$ & \\
\hline$>8$ anos & $54(44,3)$ & $53(35,3)$ & \\
\hline Não informado & $10(8,2)$ & $12(8,0)$ & \\
\hline No de consultas realizadas no local & & & 0,296 \\
\hline$\leq 10$ vezes & $68(55,7)$ & $93(62)$ & \\
\hline$>10$ vezes & $54(44,3)$ & $57(38)$ & \\
\hline Qualificação profissional (residência em saúde da família) & & & $0.003^{\#}$ \\
\hline Sim & $89(73,0)$ & $83(55,3)$ & \\
\hline Não & $33(27,0)$ & $67(44,7)$ & \\
\hline
\end{tabular}

"Teste do qui-quadrado. ${ }^{*}$ Diferença estatisticamente significativa $(\mathrm{p}<0,05)$.

modelo, como variáveis associadas com melhor avaliação da ESF, a escolaridade materna e a qualificação do profissional de saúde que atende a criança (Tabela 5).

\section{Discussão}

O presente estudo verificou que as equipes da ESF que possuem profissionais com residência em medicina de família e comunidade ou residência multiprofissional em saúde da família apresentam melhor desempenho na avaliação dos atributos da APS. A qualificação do profissional manteve-se no modelo de regressão múltipla tanto na associação com o alto escore geral da APS para todo o grupo, quanto na associação apenas entre as famílias que tinham a ESF como fonte regular de atenção.

Os resultados reafirmam a necessidade de mudanças na formação dos recursos humanos na área da saúde, especificamente para a ESF. O novo modelo assistencial parece exigir um modo de atuação profissional pautado na produção do 
Tabela 5. Modelo Ajustado do Alto Valor Geral da APS, entre as crianças que tem ESF como fonte regular de atenção à saúde. Montes Claros (MG), 2009.

\begin{tabular}{lcr}
\hline \multicolumn{1}{c}{ Variáveis } & RP (IC 95\%) & p-valor $^{*}$ \\
\hline $\begin{array}{l}\text { Escolaridade materna } \\
\quad \leq 8 \text { anos }\end{array}$ & 0,008 \\
$\quad>8$ anos & 1 & \\
Profissionais com residência & $(1,04-1,31)$ & \\
$\quad$ Não & 1 & 0,001 \\
Sim & $1,23(1,09-1,38)$ & \\
\hline
\end{tabular}

cuidado e de um novo fazer em saúde, confirmando o que foi descrito por outros autores $^{11,13,16}$. As atribuições da equipe de saúde da família são múltiplas e complexas, exigindo dos profissionais de saúde conhecimentos específicos desta área. Os dados encontrados destacam maior efetividade das equipes com residência em medicina de família e comunidade e da residência multiprofissional em saúde da família, o que pode ser atribuído à formação de profissionais mais voltadas para o desempenho das funções cotidianas da atenção primária.

Observou-se um alto índice de preferência dos cuidadores pelas equipes de saúde da família como fonte regular de atenção para o cuidado de saúde das crianças, comparado com aqueles cuidadores que referiam outros serviços. Vários estudos têm avaliado os motivos que levam a população a escolherem o local para acompanhamento de saúde, estando entre os mais referidos a proximidade da moradia e a qualidade do atendimento ${ }^{17,18}$.

Embora para o escore geral da APS não tenha sido observado um valor adequado, para as famílias que utilizavam a ESF como fonte regular de cuidado para as crianças esse escore geral foi próximo ao ideal $(6,4)$ e apresentou uma diferença estatística significante em relação aos demais serviços $(p=0,000)$. Outros estudos comprovam que a avaliação da ESF baseada em seus atributos é mais adequada quando comparadas com outros tipos de serviços ou modelos assistenciais ${ }^{8-10}$.

A análise de cada um dos atributos da APS, a partir da percepção dos cuidadores das crianças que têm a ESF como fonte regular de atenção revelou melhores escores para as equipes com melhor formação (residência) com diferenças estatisticamente significantes para os atributos de longitudinalidade $(\mathrm{p}=0,049)$, integralidade/serviços básicos $(p=0,015)$ e integralidade/serviços complementares $(\mathrm{p}=0,036)$. A análise conjunta dos atributos - escore geral - registra também que as equipes da ESF onde existem profissionais com formação específica têm escore adequado $(\geq 6,6)$ com diferença estatisticamente superior às equipes que não contam com esse profissional $(0,009)$. Estes resultados demonstram que apesar de alguns atributos ainda não apresentarem escores adequados $(\geq 6,6)$, no conjunto eles são satisfatórios. Consolida-se a idéia de que as modalidades de Residência de Família e Comunidade e as Multiprofissionais representam a oportunidade para uma reflexão organizada em torno de alternativas que permitam rever os caminhos para a formação dos profissionais, na perspectiva de um trabalho mais efetivo ${ }^{13}$.

Um dado importante no presente estudo é que os escores foram atribuídos a partir da percepção do usuário. Alguns trabalhos registram que os usuários tendem a avaliar os serviços de saúde de maneira menos favorável do que gestores ou profissionais que atuam nele ${ }^{9,10}$. É possível, portanto, que uma abordagem ampliada do processo avaliativo dos atributos da APS registre melhores escores com a inserção de novos atores sociais.

Após ajuste multivariado observou-se que os cuidadores das crianças que utilizavam a ESF como referência para atendimento à saúde da criança atribuíam melhores escores a esses locais $(\mathrm{RP}=1,22$; IC95\%=1,10;1,36), quando comparados com os cuidadores que utilizavam outros tipos de serviço. Quanto ao número de consultas, as famílias cujas crianças realizaram mais de 10 atendimentos tendem, de forma similar, a atribuir melhores escores quando comparadas com as demais ( $\mathrm{RP}=1,13$; IC95\%=1,02;1,25). Especificamente em relação à qualificação profissional, registrou-se também a percepção positiva dos cuidadores que atribuíram melhores escores para as equipes onde os profissionais possuíam a residência de medicina de família e comunidade ou multiprofissional em saúde da família $(\mathrm{RP}=1,15$; IC95\% $=1,04 ; 1,27)$.

$\mathrm{O}$ ajuste multivariado apenas para o grupo cujos cuidadores referiam à ESF como fonte regular de atenção, revelou-se que apenas a escolaridade materna maior que oito anos $(\mathrm{RP}=1,17$; IC95\% $=1,04 ; 1,31)$ e a qualificação dos profissionais em saúde da família $(\mathrm{RP}=1,23$; IC95\%= $1,09 ; 1,38)$ mostraram-se estatisticamente associados com melhor escore geral atribuído à ESF. Esse resultado também reforça a percepção positiva dos usuários em relação a uma assistência diferenciada realizada pelos profissionais mais bem qualificados. É imprescindível, portanto, resgatar a discussão sobre da provável formaçãoxos.es 
mais bastratos ctados do presente estudo estonais com resid da ESF, o n fragmentada, conteudista e com prática focada na doença, que tem ocorrido na maioria das escolas médicas e que termina por não conseguir resultados positivos na avaliação dos seus serviços de saúde ${ }^{16}$.

A literatura nacional possui poucos trabalhos que utilizam o PCATool ${ }^{7-10}$, sendo que todos eles utilizam a versão dos instrumentos elaborado por Starfield et al ${ }^{19,20}$. Estudos internacionais são bem mais frequentes e registram a importância dos aspectos abordados pelo PCATool destacando a relevância dos pais no processo de avaliação da coordenação e da continuidade do cuidado $^{21}$. Aspectos semelhantes foram observados em pesquisa no Canadá ${ }^{22}$. Os resultados do presente estudo são concordantes com os registros existentes e demonstra, de forma clara, o importante papel da qualificação dos profissionais para efetivação dos atributos da APS. Confirma-se que a residência de medicina de família e comunidade e a residência multiprofissional em saúde da família são as modalidades de pós-graduação especiais e devem, de fato, ser tomadas como "padrão-ouro" no processo para a especialização do médico e enfermeiro da ESF ${ }^{12}$.

Os dados conduzem a duas conclusões naturais. A primeira delas é de que a ESF é o espaço preferencial para a promoção da saúde infantil no âmbito sanitário. A segunda é que a residência de medicina de família e comunidade e a residência multiprofissional em saúde da família apresentam potencialidades para a transformação do modelo de atenção, a partir dos atributos da APS. Registra-se que nestas especializações as práticas de cuidado em saúde acontecem no contexto real das unidades de saúde, das famílias e da comunidade.

Algumas limitações deste estudo devem ser apontadas: a APS foi avaliada apenas na visão do usuário, o que tende a torná-la menos positiva quando comparada com avaliação feita pelos profissionais ${ }^{9,10}$; o desenho de estudo não permite determinar causalidade e sim associação entre qualificação de médicos e enfermeiros em atenção primária em saúde e qualidade da atenção primária; a pesquisa foi realizada em um único município e circunscrita a equipes da zona urbana; e, finalmente, a qualidade nas equipes de saúde foi aferida apenas a partir das ações de saúde para as crianças menores de dois anos de idade. É preciso destacar, entretanto, que a qualidade do cuidado é um resultante de uma complexa interação de fatores. Não é possível supor que um único estudo pode assumir todas as facetas da atenção à saúde. A maioria dos estudos na área apresenta recortes diferentes sobre o tema exatamente pela dificuldade de incorporar olhares tão diversos. Sobre a análise conduzida neste estudo, buscou-se valorizar o contexto, já que poucos municípios brasileiros desenvolvem programas de residência e reúnem condições favoráveis para a pesquisa tal como foi desenvolvida. É preciso destacar também que a temática abordada é de caráter universal e a literatura ainda registra importantes lacunas do conhecimento na área. Assim, os resultados aqui alcançados devem ser contribuir para novas indagações e realizações de outros estudos, no desafio de construção de modelos mais efetivos e equânimes.

\section{Colaboradores}

CDA Leão e AP Caldeira participaram igualmente de todas as etapas de elaboração do artigo. 


\section{Referências}

1. Tejada de Rivero, DA. Alma-Ata: 25 años después. Rev Perspec de Salud 2003; 8(1):2-7.

2. Conill EM. Ensaio histórico-conceitual sobre a Atenção Primária à Saúde: desafios para a organização de serviços básicos e da Estratégia Saúde da Família em centros urbanos no Brasil. Cad Saude Publica 2008; 24(Supl. 1):7-27.

3. Brasil. Ministério da Saúde (MS). Departamento de Atenção Básica. Histórico de Cobertura do PSF. Brasília: Ministério da Saúde [acessado 2009 ago 20]. Disponível em: http://dtr2004.saude.gov.br/ dab/abnumeros.php

4. Almeida MCP, Mishima SM. O desafio do trabalho em equipe na atenção à Saúde da Família: "novas autonomias" no trabalho. Interface Comun Saúde Educ 2001; 5(9):150-153.

5. Camelo SH; Angerami ELS. Formação de recursos humanos para a estratégia de saúde da família. Cienc Cuid Saúde 2008; 7(1):45-52.

6. Starfield B. Atenção Primária: equilíbrio entre necessidades de saúde, serviços e tecnologia. Brasília: UNESCO; 2002.

7. Macinko J, Almeida C, Oliveira ES, Sá PK. Organization and delivery of primary health care services in Petrópolis, Brazil. Int J Health Plann Manage 2004(4); 19:303-317.

8. Ibañez N, Rocha JSY, Viana ALA, Castro PC, Ribeiro MCSA, Forster AC, Novaes MHD, Viana ALD. Avaliação do desempenho da atenção básica no Estado de São Paulo. Cien Saude Colet 2006; 11(3): 683-703.

9. Elias PE, Ferreira CW, Alves MCG, Cohn A, Kishima V, Escrivão Júnior A, Gomes A, Bousquat A. Atenção básica em saúde; comparação entre PSF e UBS por estrato de exclusão social em São Paulo. Cien Saude Colet 2006; 11(3):633-641.

10. Van Stralen CJ, Belisário AS, Van Stralen TBS, Lima, AMD, Massote AW, Oliveira CL. Percepção dos usuários e profissionais de saúde sobre atenção básica: comparação entre unidades com e sem saúde da família na Região Centro-Oeste do Brasil. Cad Saude Publica 2008; 24(Supl. 1):148-158.

11. Costa RKS, Miranda FAN. Formação profissional no SUS: oportunidades de mudanças na perspectiva da estratégia de saúde da família. Trab Educ Saúde 2008; 6(3):503-517.

12. Campos FE, Aguiar RAT. A expansão da atenção básica nas grandes cidades e a especialização em saúde da família como estratégia para sua viabilidade. In: Brasil. Ministério da Saúde (MS). Secretaria de Gestão do Trabalho e da Educação na Saúde. Cadernos RH Saúde 3(1). Brasilia (DF): Ministério da Saúde (MS); 2006. p. 97-102.
13. Gil CRR. Formação de recursos humanos em saúde da família: paradoxos e perspectivas. Cad Saude Publica 2005; 21(2):47-52.

14. Harzheim E, Starfield B, Rajmil L, Álvarez-Dardet C, Stein AT. Consistência interna e confiabilidade da versão em português do Instrumento de Avaliação da Atenção Primária (PCATool-Brasil) para serviços de saúde infantil. Cad Saude Publica 2006; 22(8):1649-1659.

15. Oliveira MMC. Presença e extensão dos atributos da Atenção Primária à Saúde entre os serviços de Atenção Primária à Saúde em Porto Alegre: uma análise agregada [dissertação]. Porto Alegre (RS): Universidade Federal do Rio Grande do Sul; 2007.

16. Ceccim RB, Feuerwerker LCM. Mudança na graduação das profissões de saúde sob o eixo da integralidade. Cad Saude Publica 2004; 20(5):1400-1410.

17. Santos IS, Baroni RC, Minotto I, Klumb AG. Critérios de escolha de postos de saúde para acompanhamento pré-natal em Pelotas, RS. Rev Saúde Pública 2000; 34(6):603-609.

18. Ramos DD, Lima MADS. Acesso e acolhimento aos usuários em uma unidade de saúde de Porto Alegre, Rio Grande do Sul, Brasil. Cad Saude Publica 2003; 19(1):27-34.

19. Shi L, Starfield B, Jiahong X. Validating the adult primary care assessment Tool. J Fam Pract 2001; 50(2):161-175.

20. Cassady CE, Starfield B, Hurtado MP, Berk RA., Nanda JP, Friedenberg LA. Clinical Care for Children: measuring consumer experiences with primary care. Pediatrics 2000; 105(4):998-1003.

21. Co JP, Macdonald E, Yucel RM, Ferris TG. Practice variation in parental assessment of pediatric ambulatory care. Acad Pediatr; 2009; 9(1):47-52.

22. Haggerty JL, Pineault R, Beaulieu MD, Brunelle Y, Gauthier J, Goulet F, Rodrigues J. Practice Features Associated With Patient-Reported Accessibility, Continuity, and Coordination of Primary Health Care. Ann Fam Med 2008; 6(2):116-123.

Artigo apresentado em 23/10/2010

Aprovado em 13/01/2011

Versão final apresentada em 15/01/2011 
